\title{
Editorial
}

\section{Advances in Device-to-Device Communications and Networks}

\author{
Lingyang Song, ${ }^{1}$ Yan Zhang, ${ }^{2}$ Stephen Wang, ${ }^{3}$ and Cheng-Xiang Wang ${ }^{4}$ \\ ${ }^{1}$ School of Electronics Engineering and Computer Science, Peking University, Beijing 100871, China \\ ${ }^{2}$ Simula Research Laboratory, Martin Linges v 17, Fornebu, 1325 Lysaker, Norway \\ ${ }^{3}$ Telecommunications Research Laboratory, Toshiba Research Europe Ltd, Bristol BS1 4ND, UK \\ ${ }^{4}$ School of Engineering \& Physical Sciences, Heriot-Watt University, Edinburgh, UK \\ Correspondence should be addressed to Lingyang Song; lingyang.song@pku.edu.cn
}

Received 19 November 2014; Accepted 19 November 2014

Copyright (C) 2015 Lingyang Song et al. This is an open access article distributed under the Creative Commons Attribution License, which permits unrestricted use, distribution, and reproduction in any medium, provided the original work is properly cited.

Welcome to this special issue. Device-to-device (D2D) communication is a promising concept to improve user experiences and resource utilization in cellular networks, which enables two or more mobile devices in proximity to establish local links, coordinated by a base station, to perform direct data exchange. The benefits of D2D communication include ubiquitous computing and communication, traffic offloading, energy efficiency enhancement, coverage extension, and creation of new services. However, many research challenges still exist, such as analyzing the performance of these systems in different channel conditions and studying the performance with different transmission and resource management techniques.

The aim of this special issue is to address research advances that enable D2D communication in cellular networks, and report on the state-of-the-art contributions in this area. To ensure quality of this special issue, we have conducted a rigorous review process on twenty-eight submissions, from which nine papers are selected and clustered into three groups: two papers on channel coding and signal processing algorithms, four on resource management and scheduling, and three on new services and applications. Detailed overviews of the selected works are given below.

The first group addresses channel coding and signal processing algorithms on physical layer. The first paper, written by M. Dai et al., studies multidevice multicast communication via a layer of parallel relay nodes and proposes two relaying strategies, respectively, called the conventional relaying and network-coded relaying. The paper, by $\mathrm{M}$. Qian et al., presents a signal detection scheme for two-way relaying network using distributed differential space-time coding under imperfect synchronization. A realistic scenario is investigated by considering the signals transmitted from the two source nodes arriving at the relay not exactly at the same time due to the distributed nature of the nodes.

The second group mainly investigates MAC and network layer resource allocation and scheduling issues. The first of these papers, by W. Rehman et al., jointly considers resource scheduling and relay selection to improve network capacity in $60 \mathrm{GHz}$ based D2D networks. Two types of transmission scenarios are considered in wireless personal area networks, that is, intra- and intergroup. A distributed receiver based relay selection scheme is proposed for intragroup transmission and a distance based relay selection scheme for intergroup transmission. The second paper, by Q. Ou et al., proposes a distributed cooperation scheme on frequency resource sharing proposed to improve the quality of service in D2D communications underlaying cellular networks using coalition formation game. And all users have the incentive to cooperate with some others and form a competitive group to maximize the probability of obtaining their favorite spectrum resources. The third paper, by B. Guo et al., investigates the resources allocation problem in multiuser scenario and proposes a graph-based resources allocation scheme which can achieve suboptimal performance but with low computational complexity and less feedback information. The paper, by Y. He et al., proposes a joint scheduling and resource allocation scheme to improve the performance of D2D communication by taking UE rate and UE fairness into account. A Stackelberg game framework by grouping 
a macrocellular UE, a femtocellular UE, and a D2D UE to form a two-leader one-follower pair is utilized to facilitate the resource allocation problem.

The third group explores new services and applications. The first paper, by X. Luan et al., proposes a distributed joint cluster formation and resource allocation scheme for data collection in cluster-based M2M networks. A hierarchical transmission model which contains two communication phases is provided in order to utilize the advantages of cooperation. The second paper, by Y. Yin et al., presents an infinite horizon Constrained Markov Decision Process to model the sensor selection, which is able to achieve different degrees of performance tradeoff between lifetime and fairness. The simulation results show that the proposed scheduling algorithm can obtain optimal lifetime utilizing optimal policy under different degrees of constraints. The last paper, by W. Zhang et al., suggests a new chaotic stream cipher-based cryptographic scheme to achieve secure data communications over a wireless sensor network for Intelligent Transportation Systems. The proposed cryptographic scheme not only provided key negotiation and data encryption between sensor nodes in the WSN but also reduced computational costs and power consumption.

In conclusion, this issue offers a state-of-the-art view into recent advances of $\mathrm{D} 2 \mathrm{D}$ communication and networks and also attempts to tackle the research challenges particularly raised from the adoption of new technologies. This issue also offers both academic and industry appeal-the former as a basis toward future research directions and the latter toward viable industrial commercialization.

Finally, we would like to thank all the authors who have submitted their papers for consideration for publications in this issue. We are grateful to the anonymous reviewers who spent much of their precious time in reviewing all submissions. Their timely reviews and comments greatly helped us select the best papers for inclusion in this special issue. We hope you will enjoy reading the great selection of papers in this issue.

Lingyang Song Yan Zhang Stephen Wang

Cheng-Xiang Wang 

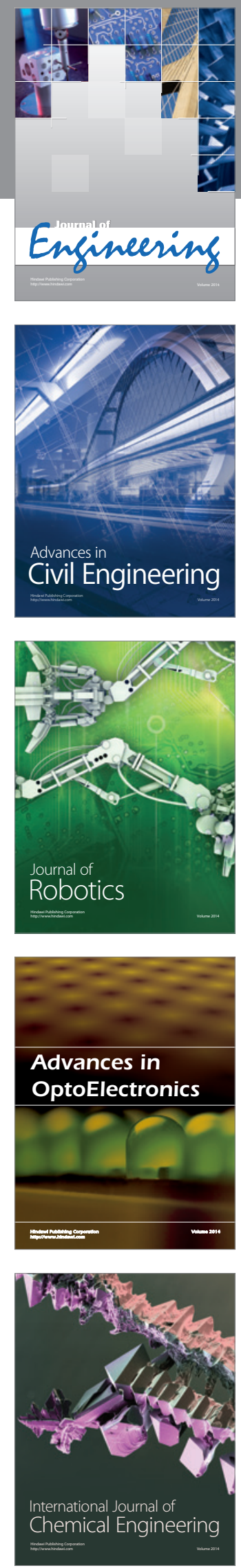

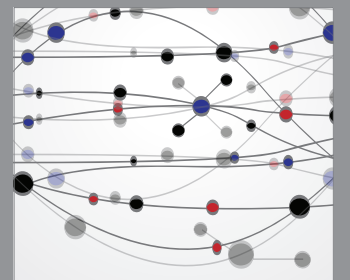

The Scientific World Journal
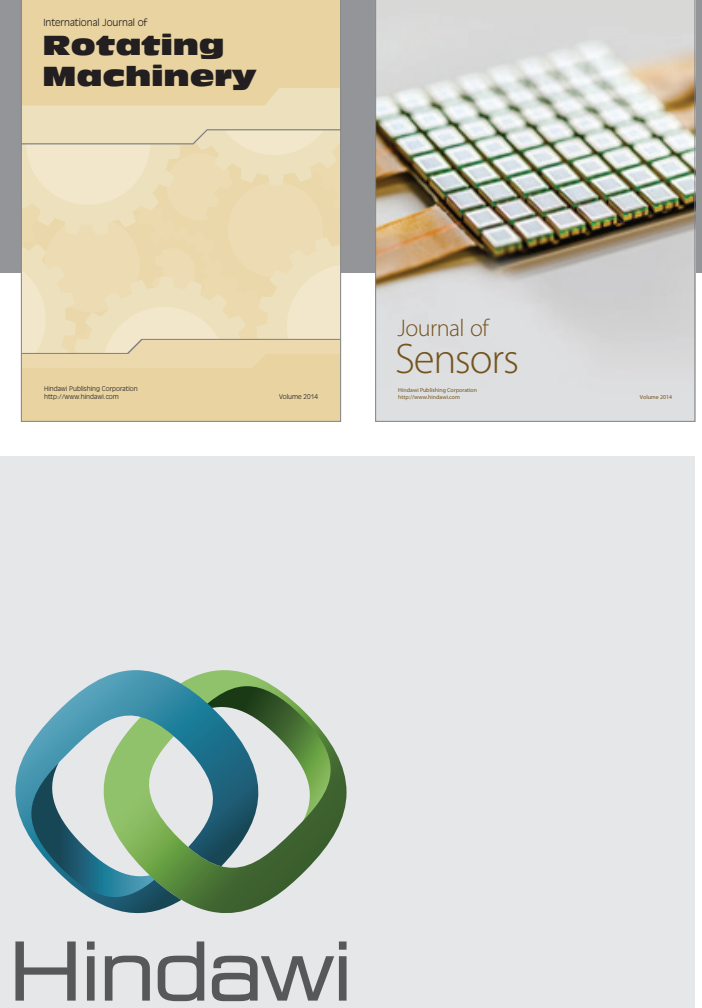

Submit your manuscripts at http://www.hindawi.com
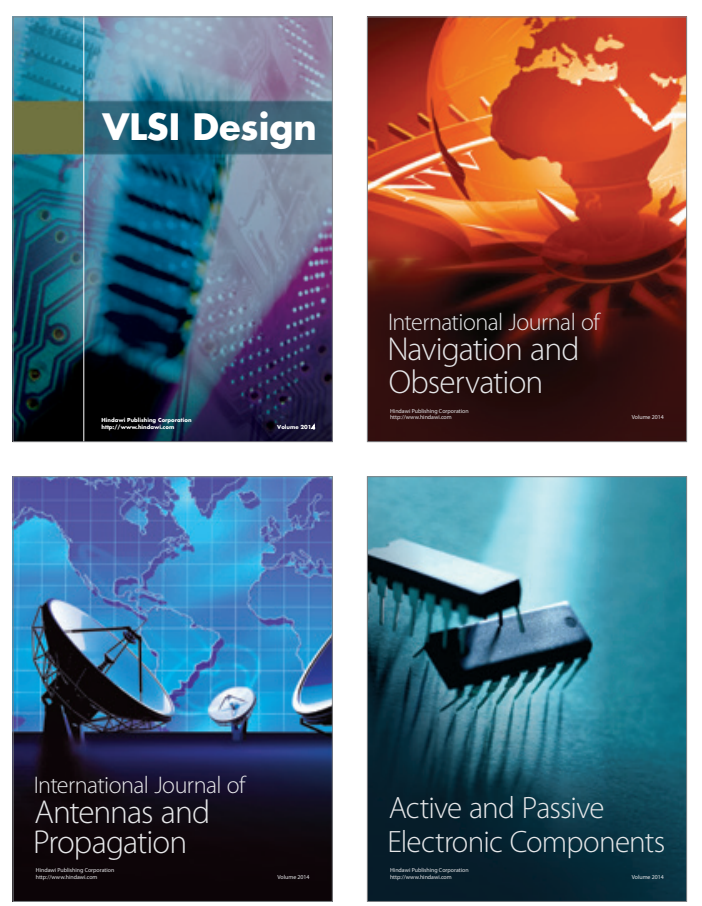
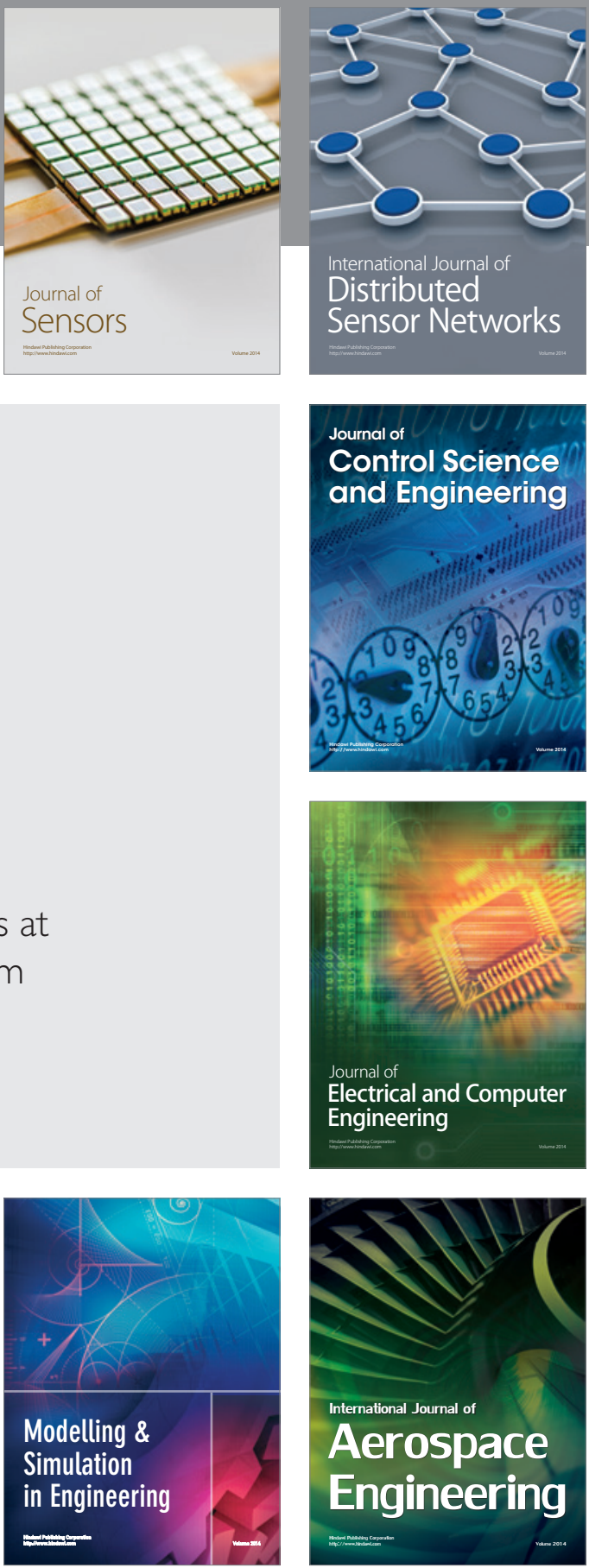

Journal of

Control Science

and Engineering
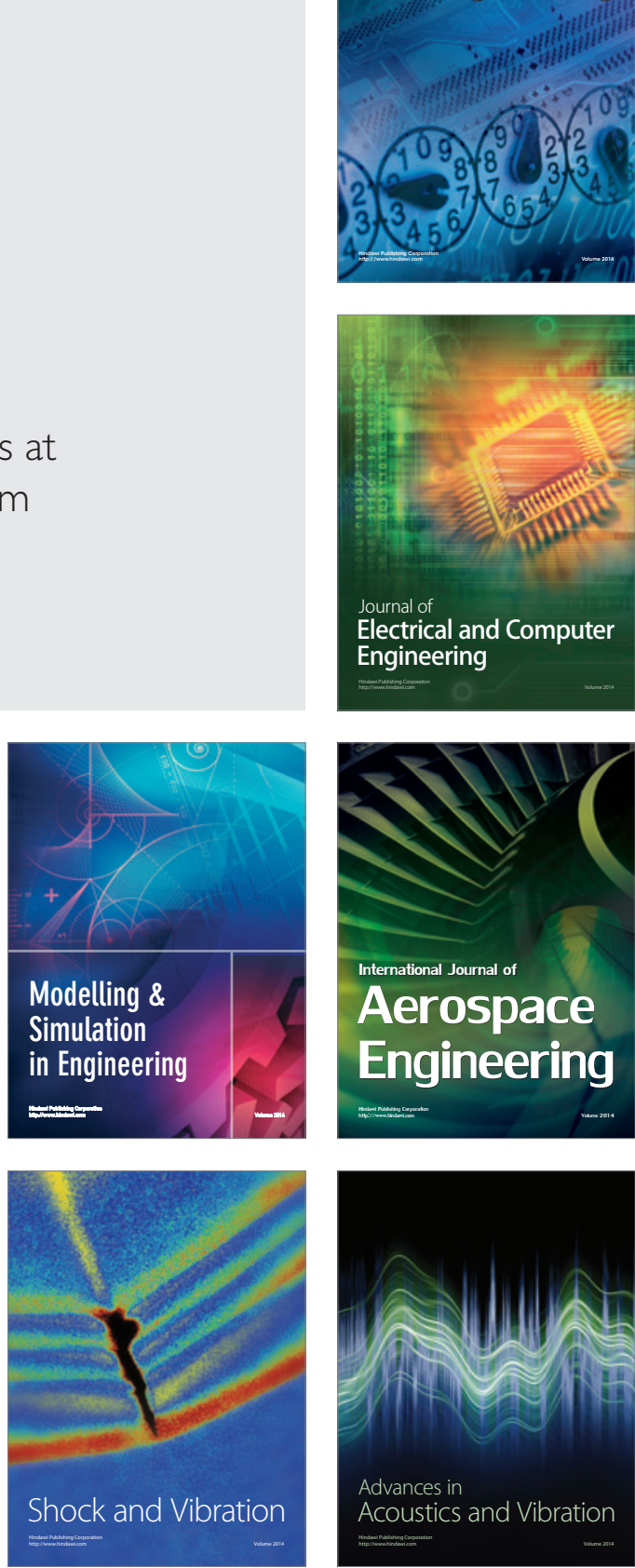